\title{
Boundary effects on the scaling of the superfluid density
}

\author{
N. Schultka ${ }^{a}$ and E. Manousakis ${ }^{b}$ \\ ${ }^{a}$ Institut für Theoretische Physik, Technische Hochschule Aachen, D-52056 Aachen, Germany
}

${ }^{b}$ Department of Physics and Center for Materials Research and Technology, Florida State University, Tallahassee, FL 32306, USA

We study numerically the influence of the substrate (boundary conditions) on the finite-size scaling properties of the superfluid density $\rho_{s}$ in superfluid films of thickness $H$ within the XY model employing the Monte Carlo method. Our results suggest that the jump $\rho_{s} H / T_{c}$ at the Kosterlitz-Thouless transition temperature $T_{c}$ depends on the boundary conditions.

In Ref.[1] we used the XY model to investigate numerically the finite-size scaling (FSS) properties of the superfluid density of superfluid films with respect to the film thickness $H$. The superfluid density $\rho_{s}$ is related to the helicity modulus $\Upsilon$ of the XY model through $\rho_{s}=(m / \hbar)^{2} \Upsilon$ where $m$ denotes the mass of the helium atom[2]. We used rather thin films up to $H=24$ and found that we were able to collapse our data for the helicity modulus onto a single curve employing the scaling expression

$$
\frac{\Upsilon(T, H) H_{e f f}}{T}=\Phi\left(t H_{e f f}^{1 / \nu}\right)
$$

where the reduced temperature $t=T / T_{\lambda}-1$ and the effective thickness $H_{\text {eff }}=H+D(D=5.8)$. Applying this idea to the experimental data of Rhee et al. 3] we achieved approximate data collapse as well $(D=0.145 \mu m)$. Note that the scaling form (ii) reduces to the conventional scaling form in the limit $H \gg D$. In Ref. [1] we argued that the increment $D$ could be understood as an effective scaling correction which takes the influence of the boundary conditions (BC) into account, i.e. vortex creation on a BC dependent length scale $l$.

In order to investigate the role of the length scale $l$ further we use the XY model on cubic lattices $L^{3}$ with periodic boundary conditions (PBC) along the $x$ - and $y$-directions and Dirichlet boundary conditions (DBC) (vanishing order parameter) along the $z$-direction and study the FSS behavior of the $x$ component of the helicity modulus with respect to $L$. (In the following we will always refer to the $x^{-}$ component of the helicity modulus as the helicity modulus.) By studying the FSS behavior of the helicity modulus of the XY model on cubic lattices with $\mathrm{DBC}$ in the $z$-direction we preserve the qualitative features of films (suppression of the helicity modulus due to DBC (cf. also [1])) but have the advantage of using "thicker" systems (up to $L=48$ ).

The hamiltonian of the XY model on a lattice is given by $\mathcal{H}=J \sum_{\langle i, j\rangle} \vec{s}_{i} \cdot \vec{s}_{j}$ where the sum runs over nearest neighbors, the pseudospins $\vec{s}_{i}=$ $\left(\cos \theta_{i}, \sin \theta_{i}\right)$ and sit on the lattice sites and $J$ sets the energy scale. The helicity modulus $\Upsilon$ is defined as in Refs. [⿴囗十 and the temperature $T$ is measured in units of $J / k_{B}$. DBC are realized on different boundary inherent length scales as follows. The layers $z=1, L$ are coupled to a spin configuration defined by

$$
\vec{s}(x, y, z)=(-1)^{\left[\frac{x}{n}\right]+\left[\frac{y}{n}\right]} \vec{s}(1,1, z), \quad z=0, L+1,
$$

with $x, y=1,2, \ldots, L$ denoting the integer coordinates of the lattice sites. The symbol $[x / n]$ means the integer part of the number $x / n$. The integer $n$ determines the linear dimension over which the spins of the boundary spin configuration are parallel. Thus, the local magnetization in the boundary $\vec{M}=\sum_{i \in A} \vec{s}_{i}$, with $A$ an area containing $2 n \times 2 n$ boundary spins, vanishes over the length scale $2 n$, i.e. the number $n$ serves as a measure for the length scale $l$ over which vortices are created by the boundary. In our Monte Carlo simulations we used $n=1$ and $n=4$ and lattice sizes $L=24,32,40,48$.

In Fig.1 we compare the FSS functions 
$\Upsilon(T, L) L / T=\Phi\left(t L^{1 / \nu}\right)(\nu=0.6705[5])$ for PBC, $n=1 \mathrm{DBC}$, and $n=4 \mathrm{DBC}$ along the $z$-direction, respectively. We find $\Phi(x)_{\mathrm{PBC}}>\Phi(x)_{\mathrm{DBC}, \mathrm{n}=1}>$ $\Phi(x) \mathrm{DBC}, \mathrm{n}=4$. This is qualitatively the same behavior as was demonstrated for the film case [1]. To see the influence of the $\mathrm{BC}$ it is instructive to consider the universal number $\Phi(0)$. We have $\Phi(0)=$ $0.505(7)(\mathrm{PBC})$ 6], $\Phi(0)=0.189(2)(\mathrm{n}=1 \mathrm{DBC})$, and $\Phi(0)=0.148(4)(\mathrm{n}=4 \mathrm{DBC})$. Combining these results and the results reported in Ref.[1] we can immediately draw conclusions for the film case. Due to the suppression of the helicity modulus we expect the Kosterlitz-Thouless transition temperatures $T_{c}(H)$ for films of thickness $H$ in the presence of DBC to satisfy the inequality $T_{c}^{n=1}(H)>T_{c}^{n=4}(H)$. Furthermore the inequality $D_{n=1}<D_{n=4}$ should hold for the increment $D$ which has to be added to the film thickness $H$ to obtain $H_{\text {eff }}$. Thus, we suppose that the larger the length scale $l$ is (or $n$ in our Monte Carlo calculations) the larger is the increment $D$ and the thicker films have to be used to verify conventional FSS in experiments.

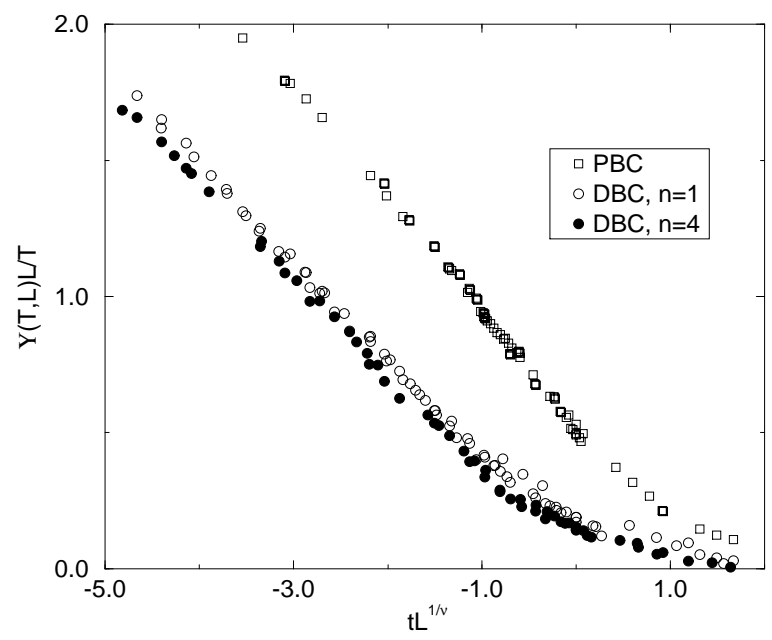

Figure 1: The scaling function $\Upsilon(T, L) L / T$ for $\mathrm{PBC}, \operatorname{DBC}(\mathrm{n}=1)$, and $\operatorname{DBC}(\mathrm{n}=4)$ along the $z_{-}^{-}$ direction.

The dependence of the value $\Phi(0)$ on the BC suggests that in the case of a film geometry the jump $\Upsilon\left(T_{c}(H), H\right) H / T_{c}(H)$ depend on the BC, too. Table 1 contains the values $\Upsilon\left(T_{c}(H), H\right) H_{\text {eff }} / T_{c}(H)$ for films with $n=1 \mathrm{DBC}$ for different film thicknesses and we see that this value seems to saturate for $H \geq 16$ at about 0.97 . Thus, for films with
$H \gg D$ we would find $\Upsilon\left(T_{c}(H), H\right) H / T_{c}(H)=0.97$ compared to the value $2 / \pi$ expected from renormalization group calculations[7] and found for films in the presence of $\mathrm{PBC}$ along the $z$-direction[B] and in experiments [9]. If we applied $n=4 \mathrm{DBC}$ along the $z$-direction of the film we would expect $\Upsilon\left(T_{c}(H), H\right) H / T_{c}(H)>0.97$.

\begin{tabular}{|l|l|}
\hline$H$ & $\Upsilon\left(T_{c}^{2 D}(H), H\right) H_{e f f} / T_{c}^{2 D}(H)$ \\
\hline 4 & $0.565(32)$ \\
8 & $0.81(17)$ \\
12 & $0.870(65)$ \\
16 & $0.974(44)$ \\
20 & $0.972(68)$ \\
\hline
\end{tabular}

Table 1: $\quad$ The jump $\Upsilon\left(T_{c}^{2 D}(H), H\right) H_{e f f} / T_{c}^{2 D}(H)$ for different thicknesses $H$.

At this point we find it quite interesting to investigate the FSS behavior of the superfluid density of superfluid films over a wide range of film thicknesses and for various substrates so that BC are realized which create vortices on different length scales. These experiments could directly test our ideas of scaling with an effective thickness for rather thin films and the dependence of the jump $\Upsilon\left(T_{c}(H), H\right) H / T_{c}(H)$ on the BC. It would be especially interesting to check these ideas using silicon as a substrate and the experimental set-up of the experiment of Rhee et al. [3].

\section{REFERENCES}

[1] N. Schultka and E. Manousakis, preprint condmat/9602085.

[2] M. E. Fisher, M. N. Barber, and D. Jasnow, Phys. Rev. B16 2032 (1977).

[3] I. Rhee, F. M. Gasparini, and D. J. Bishop, Phys. Rev. Lett. 63410 (1989).

[4] S. Teitel and C. Jayaprakash, Phys. Rev. B27 598 (1983); Y.-H. Li and S. Teitel, Phys. Rev. B40 9122 (1989).

[5] L. S. Goldner and G. Ahlers, Phys. Rev. B45 13129 (1992).

[6] N. Schultka, PhD thesis, Florida State University, (1995).

[7] D. R. Nelson and J. M. Kosterlitz, Phys. Rev. Lett. 391201 (1977).

[8] N. Schultka and E. Manousakis, Phys. Rev. B51 11712 (1995).

[9] D. J. Bishop and J. D. Reppy, Phys. Rev. Lett. 401727 (1978). 